\title{
An Overview of Approach of Social Computing and Experimental Simulation
}

\author{
Zongyuan Tan ${ }^{1, a}$ Ning Cai ${ }^{1, b^{*}}$ Jipeng Gu ${ }^{1, c}$ Jian Zhou ${ }^{1, d}$ \\ ${ }^{1}$ College of Electrical Engineering, Northwest Minzu University, Lanzhou, China \\ tanzongyuansmile@163.com, bcaining91@tsinghua.org.cn, \\ c2026321998@qq.com dzhoujianfrank@126.com
}

*The corresponding author: Cai Ning (caining91@tsinghua.org.cn)

Keywords: Social computing; Parallel model; Distributed; Module degree

\begin{abstract}
In this current article, it is endeavor to explore the possible methods that are from qualitative to quantitative and also combine computer technology to address social system interaction. A distributed paralleled model based on social computing is established to analyze different experiment data and wish to overcome the limitations of complex systems. At the same time, some simulation results will be illustrated in the follow section to further prove the feasibility of experiment.
\end{abstract}

\section{Introduction}

With the continuous enhancement of the complication of management and social problems, the computational experiments based on artificial society concept are also constantly tried, which is aimed at establishing effective model that could exactly descript social behaviors [1]. Computer experiment methods in the background of different disciplines usually adopt meta-synthesis methodology and combine computer technology, complex system and evolution theory to reappear different scenes of social management activity, which also analyze characteristic behaviors and correlation for further revealing the development rules of social complexity. At the same time, it is not only a state or a social track of simulation systems, but also could be reappeared the internal laws that can't be seen by human eyes to reveal the evolvement direction of motion of an object. Moreover, although the traditional methods of computational simulation can address a variety of complex systems, it is only a possible mean for the computer experiment methods [2,3]. In foreign research, scholars usually adopt social simulation mode for the study of social problems, which can take reasonable research for society, management and scientific problem complexity and is expected to explore the rules behind of social activity [4].

Social computing methods is used to research complex systems that are composed of society and person, which can not accurately descript their actions, and will bring great challenge to the social computing modeling based on artificial systems. Meanwhile, the study of most social problems is researched by adopting passive observation or statistical methods, which is hard to conduct active experiment for the research objects and accompany by subjective or unobservable factors to make the fact that experiment results do not possess universality [5,6]. In order to solve social computing problems, using artificial systems based on computer is to easily operate and repeat experiment, which could develop well-controlled quantitative analysis for the different impact elements of social computing [7]. Additionally, artificial systems and practice systems simultaneously operate to compose a paralleled system based on social computing, which could reciprocally compare the running process for artificial and actual objects to accomplish effective control and management of the real systems. Actually, the social computing methods based on artificial system no longer are to approach practical complex systems as the sole criterion, but is a replaceable or realizable form $[8,9]$.

The achievement of social computing is strongly associated with the paralleled systems, and it is rather significant that multiple paralleled systems address complex problems to the experiment 
efficiency [10]. Firstly, in order to effectively build and apply a paralleled system to settle social problems, it is very necessary that multiple targets, multiple solutions and evaluation systems are established to further analyze social complex systems. Secondly, referring to the mature self-adaptation control theory compiles relevant self-adaptation algorithms for building internal reflection mechanism of the paralleled systems. At the same time, using the interval rolling methods of optimization theory set up the perturbation analysis of the social paralleled systems. Finally, it is very important to achieve various combinations of patterns by combining artificial systems and actual systems, which the purpose is to analyze the similarities and differences of various systems. It is expected to simplify the complex systems by the research of the paralleled systems, which could further facilitate the development of experiment methods based on social computing.

\section{Module Degree Formulation}

The concept of module degree is first proposed by Newman, which would provide an assistance for further improving the veracity of human action and the degree of community division. The fundamental formulation can be expressed as follows:

$$
\sum_{i \in C, j \in C}\left(A_{i, j}-d_{i} d_{j} / 2 m\right)
$$

And if the community can be directly divided into $k$, the overall degree of network community can be defined as that:

$$
\sum_{l=1}^{k} \sum_{i \in C_{L}, j \in C_{l}}\left(A_{i, j}-d_{i} d_{j} / 2 m\right)
$$

Thus, the module degree formulation can be comprehensively illustrated:

$$
Q=\frac{1}{2 m} \sum_{l=1}^{k} \sum_{i \in C_{l}, j \in C_{l}}\left(A_{i, j}-d_{i} d_{j} / 2 m\right)
$$

According to the above formulation, we propose a numerical model which can be named waterfall model. It is worth remarking a fact that the social computing methods based on artificial system no longer are to approach practical complex systems as the sole criterion, but is a replaceable or realizable form. Also note that the primary aim of social computing is to explore the actual simulation results, rather than to reproduce the actual social community and human action. See Fig. 1.

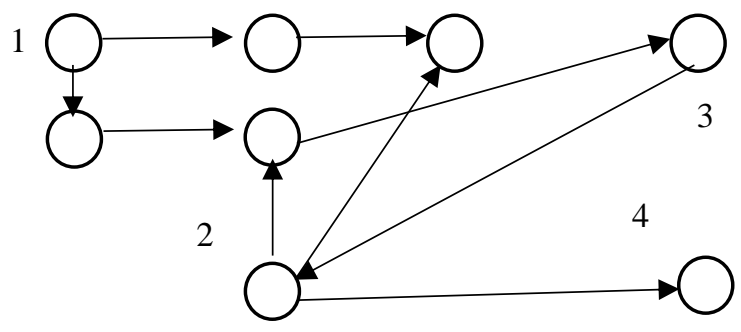

Figure 1. Waterfall model

In some sense, in particular for nonlinear social community, the methods and application of social computing have been increasingly focused by more and more related searchers. Even in some allocation tasks and modeling, social computing would be viewed as a potential tool to further computing and counting the number and degree of actual social community and complex networks. See Tab. 1 and Fig. 2. In some sense, information of actual social community can be transmitted between neurons via synapses, and the electrical activity of a given neuron can be changed by other neurons that synapse onto it. 
Table 1 Topology degree of waterfall model

\begin{tabular}{llllllll}
\hline No. & $\mathrm{N}$ & $\mathrm{L}$ & $N_{C}$ & $N_{g}(\%)$ & $\langle\mathrm{d}\rangle$ & $\lambda_{\text {in }}$ & $\lambda_{\text {out }}$ \\
\hline 1 & 187 & 324 & 0.019 & 114 & 3.144 & N/A & 1.2 \\
2 & 1540 & 1145 & 0.001 & 143 & 1.413 & N/A & N/A \\
3 & 625 & 655 & 0.003 & 414 & 1.890 & 0.9 & 0.9 \\
4 & 312 & 194 & 0.004 & 57 & 1.974 & -1.3 & N/A \\
5 & 659 & 468 & 0.002 & 136 & 5.064 & N/A & 0.8 \\
\hline
\end{tabular}

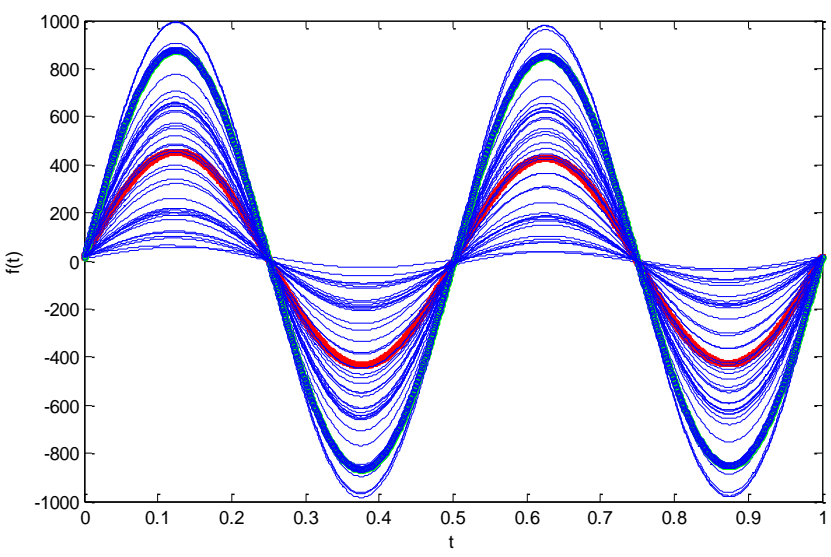

Figure 2. Simulation results of actual social community

That means it is impossible to find a proper topology degree, thus, we would attempt to simulate and experimental the social community.

$$
S O-P M I(\text { social })=\sum_{p \in p_{\Delta}} P M I-\sum_{p \in p_{\Delta}} D M I
$$

Hence, the greatest explanation is following:

$$
E_{\max }=\arg \max _{E_{i}^{\prime} \in E} P\left(E_{i} / O\right)
$$

Thus, it is customary in RMT to unfold eigenvalues $k$ through transformation

$$
\overline{\lambda_{i}}=\bar{N}\left(\lambda_{i}\right)
$$

where $\bar{N}\left(\lambda_{i}\right)=\int_{\lambda_{\min }}^{\lambda_{i}} \rho\left(\lambda^{\prime}\right) d \lambda^{\prime}$ measures the probability of an eigenvalue falling within the intervals $\left[\lambda_{\min }, \lambda_{i}\right]$. Because the function $\mathrm{N}$ is unknown, the unfolding process is implemented with the help of numerical curve fitting. Many recent modeling works focus on the neuronal synchronization in the astroglial and complex networks, also that the method and idea of the social computing would be applied into the energy power and artificial yields. As know well, target wave can occur in a reaction-diffusion system and a two-dimensional array under local Heterogeneity or external periodical forcing in local area, and the continuous emitting wave can regulate the collective behavior of the media or coupled oscillators. Indeed, spiral wave also can be generated from heterogeneity after collision between ordered waves,

The NNSD calculated from complex systems can be predicted by the Brody formula some open problems in this field such as electromagnetic radiation on electrical activities of neuron, energy consumption in neurons are presented. 


$$
p_{\beta}\left(s_{1}\right)=A s_{1}^{\beta} \exp \left(-\alpha s_{1}^{\beta+1}\right)
$$

where $\mathrm{A}$ and $\mathrm{B}$ depend on parameter $\beta$ as follows:

$$
A=(1+\beta) B
$$

and

$$
B=\left[\Gamma\left(\frac{\beta+2}{\beta+1}\right)\right]^{\beta+1}
$$

In the current note, we will point a fact that for real-world complex networks if only with noises on the communication links, such as communication networks, power networks, biological network, economic network, social network, etc. almost every network is controllable in the sense of exact controllability and stability. The analysis is also analogous to the decouplability problem for complex networks. Furthermore, some reliable neuronal circuits could be implemented for signal detection as sensors. Some biological experiments and also theoretical neuronal models have shown that single neuron exhibits rich dynamical behaviors such as periodic, social computing, simulation experiment and human action.

\section{Conclusions}

With the evolvement of social systems more and more complex, it is hard to completely use the methods of analytical reasoning analyzing the social computing problems. How to solve the practical problems of complex society have become motive power of continuous exploration of scholars, which also become a key for promoting the development of relevant field. The experiment simulation of social computing moves to a higher stage with the development of computer technology and analytical methods. Properly speaking, standardizing the computer experiment and setting relevant behavior criterion is to simulate different object for reproducing the entire process of experiment. Meanwhile, the various elements and decision parameters that affect experiment results are reasonably formulated. In addition, analyzing the consequences of computing experiment and counting correspondingly mature experiment design is to find out the rules the social complex system. Computing experiment also follows the foundational theories of experiment design that are randomization and fragmentation to guarantee validity of the experiment of social computing.

\section{References}

[1] F. Y. Wang: IEEE Intelli Syst, Vol. 26 (2011) No.1, pp.2-4.

[2] F. Y. Wang: IEEE Intelli Syst, Vol. 22 (2007) No.5, pp.65-67.

[3] N. Cai, M. J. Khan: Int. J. Control Automat. Syst., Vol. 15 (2017) No.1, pp.169-177.

[4] Y. Q. Guan, Z. J. Ji, L. Zhang and L. Wang: Int. J. Robust Nonlin. Control, DOI: 10.1002/rnc.3798.

[5] N. Cai, J. W. Cao, H. Y. Ma and C. X. Wang: Arab J Sci Eng, Vol. 39 (2014) No.3, pp. 2427-2434.

[6] Z. J. Ji, H. Lin and H. S. Yu: IEEE Trans. Automat. Control, Vol. 60 (2015) No.3, pp.781-786.

[7] N. Cai, C. Diao and M. J. Khan: Complexity, 4978613, 2017.

[8] Z. J. Ji and H. S. Yu: IEEE Trans. Cybernet., DOI: 10.1109/TCYB.2016.2549034.

[9] J. X. Xi, M. He, H. Liu and J. F. Zheng: J Franklin Inst, Vol. 353 (2016) No.16, pp.4074-4090. [10]N. Cai, M. He, Q. Wu and M. J. Khan: J. Syst. Sci. Complexity, DOI: 10.1007/s11424-017-6273-7. 